\title{
Modern Invasive Hemodynamic Assessment of Pulmonary Hypertension
}

\author{
Alberto Pagnamenta $^{\mathrm{a}-\mathrm{c}}$ Frédéric Lador ${ }^{\mathrm{c}} \mathrm{d}$ Andrea Azzola ${ }^{\mathrm{a}} \mathrm{e}$ \\ Maurice Beghetti ${ }^{d, f}$ \\ ${ }^{a}$ Department of Intensive Care Medicine, Ente Ospedaliero Cantonale (EOC), Intensive Care Units, Regional Hospital \\ of Mendrisio and Lugano, Mendrisio, Switzerland; 'b Unit of Clinical Epidemiology, Ente Ospedaliero Cantonale \\ (EOC), Bellinzona, Switzerland; ' Division of Pneumology, University of Geneva, Geneva, Switzerland; d Pulmonary \\ Hypertension Program, University of Geneva, Geneva, Switzerland; ' Pneumology, Department of Internal Medicine, \\ Regional Hospital of Lugano, Lugano, Switzerland; ${ }^{f}$ Pediatric Cardiology Unit, Children's University Hospital, \\ University of Geneva, Geneva, Switzerland
}

\section{Keywords}

Right heart catheterization - Pulmonary vascular resistance . Partitioning of pulmonary vascular resistance - Pulmonary vascular impedance $\cdot$ Right ventriculo-arterial coupling

\begin{abstract}
Since 1998 pulmonary hypertension has been clinically classified into five well-defined, distinct categories. A definitive diagnosis of pulmonary hypertension requires the invasive confirmation of an elevated mean pulmonary artery pressure of $25 \mathrm{~mm} \mathrm{Hg}$ or above during a right heart catheterization. From a hemodynamic point of view, pulmonary hypertension is classified into precapillary and postcapillary pulmonary hypertension on the basis of a pulmonary artery wedge pressure threshold value of $15 \mathrm{~mm} \mathrm{Hg}$. Pulmonary vascular resistance is better characterized by multi-point pressure/flow measurements than by single-point determination. Multi-point pulmonary vascular resistance calculation could be useful for early disease identification as well as for treatment response assessment. Occlusion analysis of the pulmonary artery pressure decay curve after balloon inflation at the tip of the pulmonary artery catheter permits locating the site of predominantly increased resistance and could be useful in differentiating proximal from distal vascu-
\end{abstract}

() 2018 S. Karger AG, Basel lopathy, especially in chronic thromboembolic pulmonary hypertension. The pulsatile hydraulic load of the pulmonary circulation can be better appreciated by pulmonary vascular impedance or via the resistance-compliance relationship than by means of pulmonary vascular resistance. Determination of right ventriculo-arterial coupling permits assessing the impact of an elevated afterload on right ventricular function, which ultimately determines the symptoms and prognosis of patients with pulmonary hypertension. The clinical utility of combining different invasive hemodynamic approaches is still uncertain and remains to be determined.

(c) 2018 S. Karger AG, Basel

\section{Introduction}

Pulmonary hypertension $(\mathrm{PH})$ is a hemodynamic and pathophysiological condition characterized by the presence of a resting mean pulmonary artery pressure (PAPm) of $\geq 25 \mathrm{~mm} \mathrm{Hg}$ as assessed by right heart catheterization (RHC) [1]. In the general population, the exact prevalence of $\mathrm{PH}$ is unknown; however, it is presumed to represent the third leading cardiovascular condition after systemic hypertension and coronary artery disease [2].

\section{KARGER}

E-Mail karger@karger.com

www.karger.com/res
Alberto Pagnamenta, MD, MSc

Intensive Care Unit, Regional Hospital of Mendrisio

Via Turconi 23

CH-6850 Mendrisio (Switzerland)

E-Mail alberto.pagnamenta@ eoc.ch 
Table 1. Clinical classification of pulmonary hypertension

1. Pulmonary arterial hypertension $(\mathrm{PAH})$

1.1 Idiopathic $\mathrm{PAH}$

1.2 Heritable PAH

1.2.1 BMPR2

1.2.2 ALK-1, ENG, SMAD9, CAV1, KCNK3

1.2.3 Unknown

1.3 Drug and toxin induced

1.4 Associated with:

1.4.1 Connective tissue disease

1.4.2 Human immunodeficiency virus infection

1.4.3 Portal hypertension

1.4.4 Congenital heart diseases

1.4.5 Schistosomiasis

1' Pulmonary veno-occlusive diseases and/or pulmonary capillary hemangiomatosis

1" Persistent pulmonary hypertension ( $\mathrm{PH})$ of the newborn

2. $\mathrm{PH}$ due to left heart diseases

2.1 Left ventricular systolic dysfunction

2.2 Left ventricular diastolic dysfunction

2.3 Valvular disease

2.4 Congenital/acquired left heart inflow/outflow tract obstruction and congenital cardiomyopathies

3. PH due to lung diseases and/or hypoxia

3.1 Chronic obstructive pulmonary disease

3.2 Interstitial lung disease

3.3 Other pulmonary diseases with a mixed restrictive and obstructive pattern

$3.4 \quad$ Sleep-disordered breathing

3.5 Alveolar hypoventilation disorders

3.6 Chronic exposure to high altitude

3.7 Developmental lung diseases

4. Chronic thromboembolic $\mathrm{PH}$

5. PH with unclear multifactorial mechanisms

5.1 Hematologic disorders: chronic hemolytic anemia, myeloproliferative disorders, splenectomy

5.2 Systemic disorders: sarcoidosis, pulmonary histiocytosis, lymphangioleiomyomatosis

5.3 Metabolic disorders: glycogen-storage disease, Gaucher disease, thyroid disorders

5.4 Others: tumoral obstruction, fibrosing mediastinitis, chronic renal failure, segmental $\mathrm{PH}$
The first clinical classification of $\mathrm{PH}$ proposed in 1973 at the World Symposium of PH (WSPH) was based on only two categories: primary $\mathrm{PH}$ and secondary $\mathrm{PH}$ [3]. The clinical classification has thereafter been refined at subsequent WSPH, with the most recently updated version proposed during the 5th WSPH held in Nice, France, in 2013 (presented in Table 1) [4]. Nowadays, PH is classified into five well-defined, distinct major clinical categories. Each category contains multiple clinical conditions according to similarities in their clinical presentation, pathophysiological characteristics, and treatment options. Furthermore, congenital/acquired left heart inflow or outflow obstructive lesions and congenital cardiomyopathies have been added to $\mathrm{PH}$ due to the group of left heart diseases (LHD), creating a common classification for both pediatric and adult patients.

Comparative epidemiological data on the prevalence of the different $\mathrm{PH}$ categories are lacking, but realistically LHD represents the most common cause of $\mathrm{PH}$ in highincome countries, including both LHD with a preserved ejection fraction and LHD with a reduced ejection fraction $[5,6]$. Chronic lung diseases and/or hypoxia account for the second most prevalent etiology of $\mathrm{PH}$ [7]. The cumulative incidence of chronic thromboembolic $\mathrm{PH}$ (CTEPH) has been estimated to lie between 0.1 and 9.1\% after an acute pulmonary embolism, but a significant number of subjects develop CTEPH without a symptomatic event of pulmonary embolism [8]. Pulmonary arterial hypertension $(\mathrm{PAH})$ remains a rare disease with an estimated prevalence of about 5-15 cases per 1 million adults [9], and over the last decades a changed $\mathrm{PAH}$ phenotype has emerged (regarding age, sex, comorbidities, and survival) [10]. Although it is the less frequent form of $\mathrm{PH}$, paradoxically $\mathrm{PAH}$ is the one that has been more extensively investigated, whereas fewer data are available on the other PH etiologies.

This narrative review aims to provide the current state of knowledge about invasive hemodynamic evaluation of $\mathrm{PH}$. Furthermore, we describe some recent advances in invasive assessment, emphasizing their advantages and their limitations as well as their potential applicability in daily clinical practice.

\section{Hemodynamic Definitions and Pulmonary Vascular Resistance}

The normal resting PAPm $( \pm \mathrm{SD})$ is approximately $14 \pm 3 \mathrm{~mm} \mathrm{Hg}$ with an upper limit of $20 \mathrm{~mm} \mathrm{Hg}$ [11] Given the unclear prognostic and treatment implications, 
the term "borderline $\mathrm{PH}$ " for subjects with a resting PAPm between 21 and $24 \mathrm{~mm} \mathrm{Hg}$ has been abandoned [1]. However, these subjects should be carefully followed up, especially those with connective tissue disease, as well as family members of patients with idiopathic PAH (iPAH) and heritable PAH given the possible future development of manifest PAH [1]. This statement has recently been further supported by the observation that these subjects showed a very high prevalence $(86 \%)$ of abnormal exercise-induced pulmonary vascular responses and a reduced exercise capacity compared with subjects with normal PAP values [12]. Before the 4th WSPH in 2008, PH had also been defined by a PAPm $>30 \mathrm{~mm}$ $\mathrm{Hg}$ at exercise [13]. This additional criterion was thereafter eliminated and introduced no more, because exercise has not been clearly specified and standardized and the exercise PAPm varies with age, rendering it impossible to find a clear cutoff value for exercise-induced $\mathrm{PH}[1,11]$.

However, recently this has been reevaluated, and the following criteria for the diagnosis of exercise-induced PH emerged: PAPm $>30 \mathrm{~mm} \mathrm{Hg}$ with a total pulmonary vascular resistance $($ total $\mathrm{PVR}=\mathrm{PAPm} / \mathrm{CO})>3 \mathrm{Wood}$ units (WU) at maximum exercise [14]. The inflow pressure of the pulmonary circulation is the PAPm and the outflow pressure is the left atrial pressure (LAP). In daily clinical practice, pulmonary vascular pressures and flow are typically measured with a fluid-filled thermodilution pulmonary artery catheter (PAC). The PAC cannot directly measure LAP, but the latter can be derived from a "wedged" PAP (PAWP) after balloon inflation at the tip of the PAC. In a large-scale clinical study with sequential measurements of PAWP and LAP estimated by left ventricular end-diastolic pressure (LVEDP) during left heart catheterization, the estimation of LAP by PAWP measurement was found to be accurate but imprecise [15]. Furthermore, among patients with $\mathrm{PH}$, roughly half of those with a PAWP $\leq 15 \mathrm{~mm} \mathrm{Hg}$ had an LVEDP $>15 \mathrm{~mm}$ $\mathrm{Hg}$, suggesting that PAWP frequently underestimates LAP; however, invasive diagnostic criteria for left heart failure with a preserved ejection fraction require a PAWP $>12 \mathrm{~mm} \mathrm{Hg}$ or an LVEDP $>16 \mathrm{~mm} \mathrm{Hg}$ [16].

$\mathrm{PH}$ is hemodynamically classified into pre- and postcapillary based on a threshold value for the PAWP of 15 $\mathrm{mm} \mathrm{Hg}$ [2]. Postcapillary PH is characterized by a PAPm $\geq 25 \mathrm{~mm} \mathrm{Hg}$ and a PAWP $>15 \mathrm{~mm} \mathrm{Hg}$ and occurs as a consequence of LHD [5]. Postcapillary PH is further subclassified on the basis of the diastolic pressure gradient (DPG), defined as the difference between diastolic PAP and PAWP (DPG = PAPd - PAWP), and of the PVR into (1) isolated postcapillary $\mathrm{PH}$ (PAWP $>15 \mathrm{~mm} \mathrm{Hg}$, DPG

Pulmonary Hypertension
$<7 \mathrm{~mm} \mathrm{Hg}$, and PVR $\leq 3 \mathrm{WU}$ ) and (2) combined postand precapillary $\mathrm{PH}$ (PAWP $>15 \mathrm{~mm} \mathrm{Hg}$, DPG $\geq 7 \mathrm{~mm}$ $\mathrm{Hg}$, and PVR $>3 \mathrm{WU}$ ) [2].

Proper use of RHC, especially regarding the standardization of PAWP measurements (e.g., value acquisition at the end of expiration, and zero levelling of the pressure transducer at the midthoracic line in supine position), is of central importance in hemodynamic $\mathrm{PH}$ assessment and classification, with direct consequences for treatment [1]. LeVarge et al. [17] reported that almost one-third of patients with a phenotype of precapillary $\mathrm{PH}$ without clinical or echocardiographic evidence of left ventricular (LV) dysfunction had an end-expiratory PAWP $>15 \mathrm{~mm}$ $\mathrm{Hg}$, especially obese and patients with obstructive pulmonary disease, likely as a consequence of a spontaneous positive end-expiratory intrathoracic pressure. Routine left heart catheterization is currently not recommended for $\mathrm{PH}$ evaluation, but by echocardiographic signs of LV dysfunction (systolic and/or diastolic) or by risk factors for coronary heart disease or heart failure with a preserved ejection fraction, it should be carefully considered [2].

PVR is not part of the general definition of $\mathrm{PH}$, but nowadays PVR is included in the hemodynamic definition of PAH as follows: PAPm $\geq 25 \mathrm{~mm} \mathrm{Hg}$, PAWP $\leq 15$ $\mathrm{mm} \mathrm{Hg}$, and PVR $>3 \mathrm{WU}$ in the absence of other precapillary forms (chronic lung diseases, CTEPH, and other rare diseases) $[1,2]$. Moreover, PVR is useful for the subclassification of postcapillary $\mathrm{PH}$, as previously mentioned. PVR can be reasonably calculated as the ratio of pressure to flow (Q) in accordance with Ohm's electrical law as follows:

$$
\mathrm{PVR}=(\mathrm{PAPm}-\mathrm{LAP}) \mathrm{Q}^{-1} \text {. }
$$

This equation assumes a linear relationship between pressure and $\mathrm{Q}$, and an extrapolated pressure intercept at zero $\mathrm{Q}$ crossing the origin. When these assumptions are satisfied, PVR remains constant with an increasing Q (Fig. 1, A curve) [18]. This is the case in West's lung zone III in healthy well-oxygenated lungs. However, in hypoxic pulmonary vasoconstriction as well as in several heart and respiratory diseases, the outflow pressure of the pulmonary circulation is not the LAP, and PVR is increased. In these circumstances, the effective downstream pressure of the pulmonary circulation is a closing pressure higher than the LAP, and therefore the LAP becomes an irrelevant pressure to $\mathrm{Q}$, like the height of a waterfall. Diseases associated with increased pulmonary vascular tone and/or alveolar pressure show an increased extrapolated PAP intercept at zero flow. 


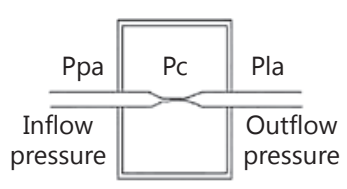

PVR $=($ Ppa $-\cdot$ Pla $) / Q$

A: Pla $>$ PC

$\mathrm{B}: \mathrm{PC}>\mathrm{Pla}$

$1 \rightarrow 2$ : vasoconstriction

$1 \rightarrow 3$ : no change in tone
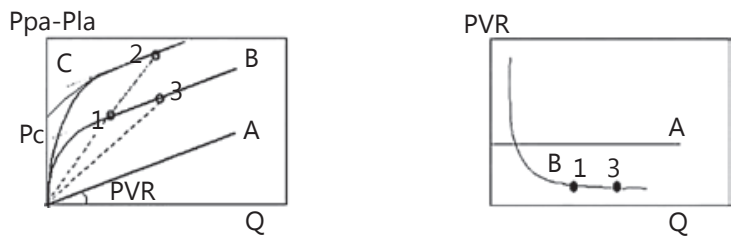

Fig. 1. Starling resistor model for explaining the concept of closing pressure within a circulatory system. Flow $(\mathrm{Q})$ is determined by the gradient between mean pulmonary artery pressure $(\mathrm{Ppa})$ and an outflow pressure which is either the closing pressure $(\mathrm{Pc})$ or the left atrial pressure (Pla). When $\mathrm{Pla}>\mathrm{Pc}$, the $(\mathrm{Ppa}-\mathrm{Pla}) / \mathrm{Q}$ relationship crosses the origin (A curve) and pulmonary vascular resistance (PVR) is constant. When $\mathrm{Pc}>\mathrm{Pla}$, the $(\mathrm{Ppa}-\mathrm{Pla}) / \mathrm{Q}$ relationship has a positive pressure intercept ( $\mathrm{B}$ and $\mathrm{C}$ curves) and PVR decreases curvilinearly with increasing $\mathrm{Q}$. Possible misleading PVR calculations: PVR, the slope of (Ppa - Pla)/Q remains unchanged in the presence of vasoconstriction (from 1 to 2 ) or decreased (from 1 to 3 ) with no change in the functional state of the pulmonary circulation (unchanged pressure/flow line). Adapted from Naeije [18] with permission from the publisher.

The clinically postulated increased closing pressure has been nicely demonstrated in an experimental intact animal model of acute lung injury, where an increased closing pressure (higher than the LAP) became the effective outflow pressure of the pulmonary circulation only after inducing an acute lung injury [19]. The closing pressure is called like that because when the intraluminal pressure drops below the closing pressure value, the pulmonary vessels collapse and the flow ceases. In accordance with the Starling resistor model of pulmonary circulation, a single-point pressure/flow determination (conventional PVR calculation) is unable to discriminate between active tone-dependent and passive flow-dependent PAP changes, and PVR can therefore be misleading in assessing the functional state of the pulmonary circulation, as shown in Figure 1 (B and C curves) [18]. To overcome this relevant inherent limitation, PAP should be measured at different levels of $\mathrm{Q}$, which permits generating multi-point pressure-flow curves. The slope of these pressure-flow coordinates defines the incremental PVR (different from the single-point PVR calculation), and the intercept at zero Q defines the effective outflow pressure of the pulmonary circulation [18]. Dobutamine can be used to increase Q; at doses up to $10 \mu \mathrm{g} \mathrm{kg}^{-1} \mathrm{~min}^{-1}$, it has no intrinsic pulmonary vasomotor properties, and therefore it can be properly used as a generator of multi-point pressure/flow plots [20].

The clinical utility of multi-point PVR determination was previously shown in patients with $\mathrm{PAH}$ in whom the resting pulmonary hemodynamic value remains unchanged (constant single-point PVR calculation) under $\mathrm{PAH}$-targeted therapy despite an increased exercise capacity as assessed by the 6 -min walk distance. In contrast, multi-point PVR significantly decreased under therapy, suggesting a predominately vasodilatory effect of the drugs $[21,22]$. These two studies indicate that "stress" pulmonary hemodynamics are more sensitive than resting hemodynamics in capturing treatment responses in PAH patients. Recently, Lau et al. [23] noninvasively assessed the functional state of pulmonary circulation using dobutamine stress echocardiography, and they found that PAH patients showed a significantly elevated incremental PVR when compared to matched healthy control subjects. Furthermore, NYHA functional class was significantly associated with dobutamine-induced PAPm Q slope but not with resting total PVR.

In conclusion, stress testing of the pulmonary circulation by generation of multipoint pressure-flow coordinates instead of single-point PVR calculation can potentially be useful for early PAH identification in at-risk populations with normal resting pulmonary hemodynamics (e.g., patients with connective tissue disease, and family members of patients with iPAH or heritable PAH), as well as for the prognosis and monitoring of treatment effects in PAH. It could also permit identifying and thereafter defining stress-induced hemodynamic treatment goals in order to better adapt medical treatment and probably to start combination therapy at an early stage. These promising preliminary results require further validation in larger multicenter clinical trials.

\section{Partitioning of PVR}

A bedside estimation of the effective pulmonary capillary pressure (Pcap) can be obtained by analysis of the PAP decay curve after a single pulmonary artery occlusion following balloon inflation at the tip of the PAC [24]. The typical PAP decay curve shows a rapid pressure decrease (filling of the capillary compartment from the arterial compartment) followed by a slower pressure drop 
(emptying of the capillary compartment into the venous one), with an inflection point in between, as shown in Figure 2 [25]. Several methods based on less or more complex mathematical analyses of the PAP decay curve have been proposed in order to estimate Pcap. The dual exponential fitting procedure seems to be the most reliable, even though uncertainty about the overlapping of arterial and capillary venous compartments may persist in case of remodeling of the smallest arterioles [24]. Assessment of Pcap allows estimating the longitudinal distribution of resistances all along the pulmonary circulation. The arterial component of PVR is calculated as (PAPm - Pcap) $\mathrm{Q}^{-1}$ and expressed as the percentage of PVR calculated as (PAPm - PAWP) $\mathrm{Q}^{-1}$.

The clinical usefulness of the partitioning of PVR has previously been shown in patients with $\mathrm{PH}$. The arterial component of PVR is increased in CTEPH and decreased in pulmonary veno-occlusive disease as compared to $\mathrm{PAH}$ [25]. This technique may be helpful in identifying the site of predominantly increased resistance, but it does not allow any clear discrimination between the different $\mathrm{PH}$ groups on an individual basis [26]. In select patients, $\mathrm{CTEPH}$ is a disease potentially curable by pulmonary thromboendarterectomy [8]. Patients with lower preoperative upstream resistance (the lower arterial component of PVR) have been identified to be at an increased risk for persistent $\mathrm{PH}$ and death after pulmonary thromboendarterectomy due to possible concomitant distal small-vessel disease [27]. In a diagnostic test study, Toshner et al. [28] found a fair-to-good ROC curve of upstream PVR (AUC 0.75; $p<0.001$ ) for discriminating operable from inoperable CTEPH, but unfortunately in up to $20 \%$ of the subjects an accurate PAP decay curve was not obtainable, and in a subgroup of patients the Pcap measured in different lung lobes yielded rather different values, suggesting a heterogeneous distribution of the disease.

While occlusion pressure analysis has been validated and can be easily performed during routine bedside RHC, further studies are still required before it will become part of routine invasive assessment of $\mathrm{PH}$ given the abovementioned limitations to this technique. Nowadays, there is no gold standard method for assessing the operability of CTEPH patients, and consequently the evaluation process remains complex [8]. In daily clinical practice, a multidisciplinary team consisting of an experienced pulmonary endarterectomy surgeon, radiologists, and CTEPH physicians evaluates the best treatment option. Definitive inoperability should be assessed by at least two independent experienced pulmonary endarterectomy surgeons

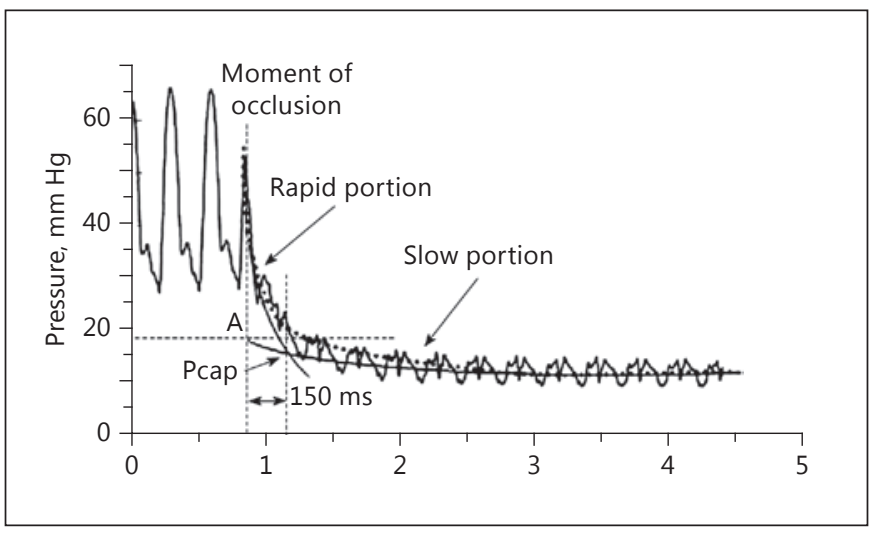

Fig. 2. Biexponential curve fitting for estimation of pulmonary capillary pressure (Pcap) by intersection of the fast and the slow component of the pressure decay curve, or by extrapolation of the exponential fitting of the slow component of the pressure decay curve to the moment of occlusion. Reproduced form Souza et al. [25] (Open Access; permission not required).

[8]. Analysis of the PAP decay curve by the occlusion method seems a promising technique for preoperative risk assessment and for patient selection for medical therapy. However, this approach should be further implemented in order to better discriminate proximal vasculopathy from peripheral involvement in CTEPH. Moreover, the occlusion method can be combined with calculation of the resistance-compliance (RC) time (see below for details) in order to better identify sites of increased resistance. A clinical prediction rule based on imaging techniques, analysis of the PAP decay curve by the occlusion method, and calculation of the RC time is potentially helpful in assessing CTEPH operability, but it still requires prospective validation.

\section{Continuous versus Pulsatile Pulmonary Hemodynamics}

An RHC with measurements of pressures and Q and PVR calculation is recommended as the reference method for diagnostic confirmation of suspected PAH, evaluation of disease severity, and determination of prognosis and response to treatment $[1,2]$. The fluid-filled thermodilution PAC provides satisfactory determinations of only mean pressure and mean flow $[29,30]$. This "steadyflow" approach imposes a simplification that neglects the natural pulsatility of the pulmonary circulation [31]. Even if PVR is optimally estimated from multi-point 
Fig. 3. Pulmonary vascular impedance (PVZ) spectrum. a Instantaneous pulmonary flow and pressure measurements. b PVZ spectrum following decomposition of the pressure and flow waves into their respective harmonics. $\mathrm{PVZ}$ as the ratio of pressure harmonics to flow harmonics, with estimated characteristic impedance $\left(Z_{c}\right)$ at high frequencies. $c Z_{c}$ estimation in the time domain as the slope of the linearized early PAPs/Q curve by Dujardin. Ppa, mean pulmonary artery pressure. Reproduced from Naeije [35] with permission from the publisher.

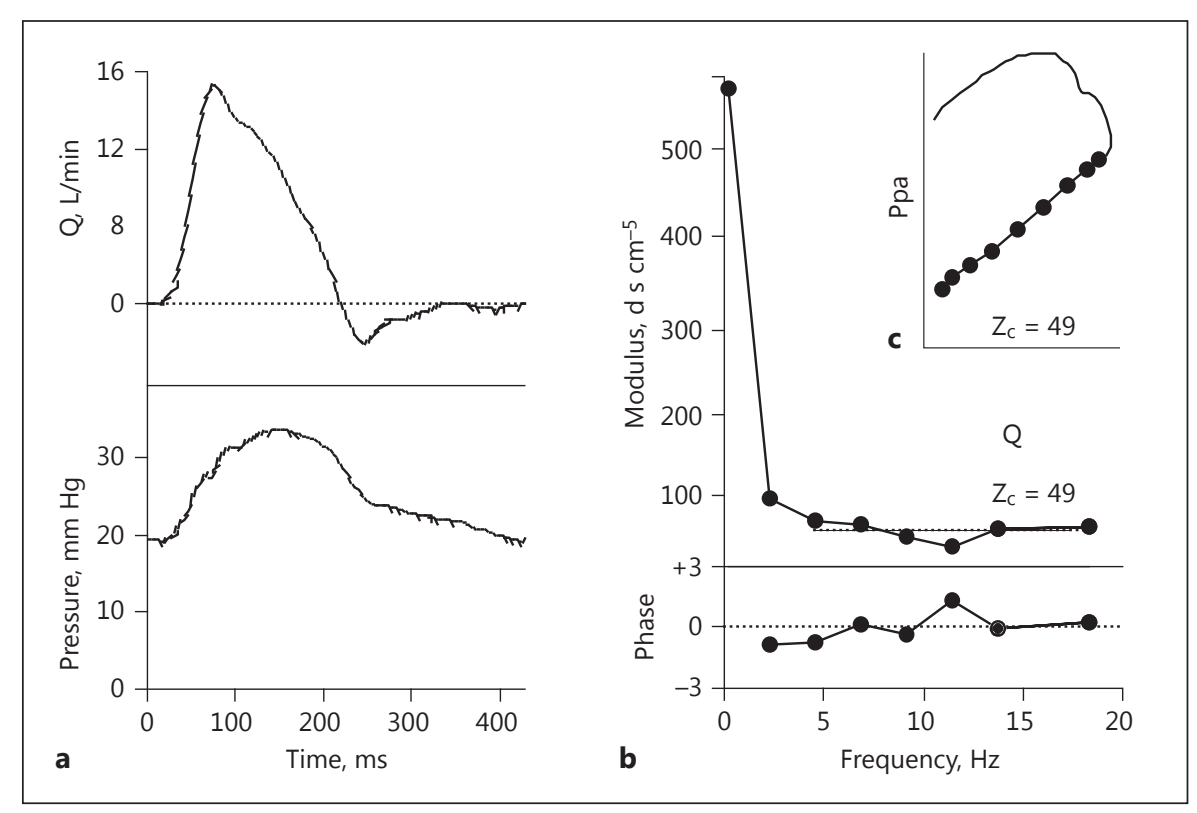

pressure-flow plots, the use of PVR as a measure of the opposition to flow presented to the right ventricle (RV) ignores two important characteristics in an oscillatory system, i.e., pulmonary vascular compliance and wave reflection, and, consequently, PVR underestimates the effective RV afterload [32].

This is a relevant limitation, since it has recently been realized to a greater extent that $\mathrm{RV}$ function is a major determinant of functional state, exercise capacity, and prognosis in $\mathrm{PH}$ [33]. The most accurate quantification of RV afterload available is obtainable by computation of pulmonary vascular impedance (PVZ). PVZ is determined by a dynamic interplay between PVR, vascular compliance, reflected waves, and blood inertance during RV ejection [31,34]. Assessment of pulsatile pulmonary hemodynamics unfortunately requires recording instantaneous PAP and Q waves simultaneously in the frequency domain rather than in the time domain. Fourier analysis permits decomposing pressure and flow waves into their respective series of harmonics at multiples of the heart rate frequency [31]. The ratio of pressure harmonics to flow harmonics defines PVZ, and unlike PVR it cannot be expressed as a single numerical value but is displayed as a graphic spectrum of pressure/flow moduli and the phase angle, both as a function of frequency, as shown in Figure 3 [35]. Normally, the PVZ spectrum decreases rapidly from a high value at $0 \mathrm{~Hz}$ to a first minimum at $2-4$ $\mathrm{Hz}$ and increases again to a first maximum at $6-8 \mathrm{~Hz}$ followed by smaller fluctuations at higher frequencies [31,
35]. $\mathrm{PVZ}$ at $0 \mathrm{~Hz}\left(\mathrm{PVZ}_{0}\right)$, called total PVR (PVR with omission of PAWP from the calculation) is the ratio of PAPm to mean Q. At high frequencies, impedance values become constant, and this PVZ, named "characteristic impedance" $\left(\mathrm{PVZ}_{\mathrm{C}}\right)$, reflects the ratio of inertial elements to compliant elements in proximal pulmonary arteries. Any increase in $\mathrm{PVZ}_{\mathrm{C}}$ suggests a decline in pulmonary vascular compliance. Resistance vessels influence the low-frequency PVZ spectrum, intermediate pulmonary vessels influence the mid-range-frequency PVZ spectrum, and proximal vessels influence the high-frequency PVZ spectrum [34].

PVZ is not commonly investigated at the bedside. Computation of PVZ needs an invasive approach with high-fidelity technology, which is too demanding and difficult to integrate into daily clinical practice; consequently there are only few reports on PVZ calculation in humans $[31,34]$. In a recent validation study, a simple bedside approach (PAC and transthoracic echocardiography for pressure and flow determination, respectively) was compared with the high-fidelity reference method (highfidelity Millar catheter and ultrasonic flow probe) using linear regression and Bland-Altman agreement analyses. The results confirmed that it is possible to derive valid estimates of pulsatile pulmonary hemodynamics with the simple bedside tools commonly employed in daily clinical practice [36]. Despite these encouraging findings, the pulsatile assessment of pulmonary circulation using PVZ computation is unlikely to become part of routine clinical 
practice due to the time-consuming nature of the technique and the complexity of hemodynamic data acquisition and analysis.

The simplest clinical estimate of pulmonary vascular compliance $(\mathrm{Ca})$ is the ratio between stroke volume and pulse pressure ( $\mathrm{PP}=\mathrm{PAPs}-\mathrm{PAPd}$ ) [37]. The product of PVR multiplied by Ca defines the RC time. PVR and Ca follow a highly predictable inverse hyperbolic relationship: any change in PVR implies an inverse change in $\mathrm{Ca}$, permitting the estimation of Ca from PVR using a simple formula. This finding was confirmed in subjects without $\mathrm{PH}$ and over a wide variety of forms, severities, and treatments of $\mathrm{PH}$ with a corresponding near-constant $\mathrm{RC}$ time $[38,39]$. An important consequence of pulmonary RC dependence is the proportionality between PP and PAPm, which implies that the RV pulsatile load remains a constant fraction of total hydraulic power independently of PAP values [40]. Knowing steady power as the product of mean pressure and mean flow, RV total hydraulic power (RV afterload) can be easily calculated: total power $=1.33 \times($ PAPm $\times$ mean $Q)$. This estimation of RV afterload has the great advantage over PVZ computation of not requiring the recording of instantaneous pressure and flow signals.

This simple RV afterload calculation is potentially useful for evaluating the impact of the different $\mathrm{PH}$ forms on $\mathrm{RV}$ function. Exceptions to the consistent RC relationship were observed in patients with proximal CTEPH and in patients with elevated pulmonary venous pressure. In a large retrospective analysis, $\mathrm{RC}$ times were lowest in proximal CTEPH patients as compared to patients with distal CTEPH and patients with iPAH after adjustment for covariates [41]. These findings were also observed in an experimental model of operable CTEPH as compared to an experimental model of PAH, suggesting that the location of increased resistance affects the time constant of pulmonary circulation probably through altered wave reflection [36]. In a large clinical database, patients with chronic left heart failure and elevated PAWP $(\geq 20 \mathrm{~mm}$ $\mathrm{Hg}$ ) showed a shortened RC time, suggesting an increased pulsatile load [42]. This interesting result possibly highlights the previously underestimated deleterious effects of postcapillary $\mathrm{PH}$ on RV function.

Although valid estimates of pulsatile pulmonary hemodynamics can be obtained during routine RHC, PVZ computation and interpretation remain difficult to integrate into daily clinical practice, and this technique will probably remain confined to the experimental setting. On the other hand, $\mathrm{RC}$ dependence allows assessing effective $\mathrm{RV}$ afterload using a simple algebraic formula. Furthermore, RC time calculation can be combined with analysis

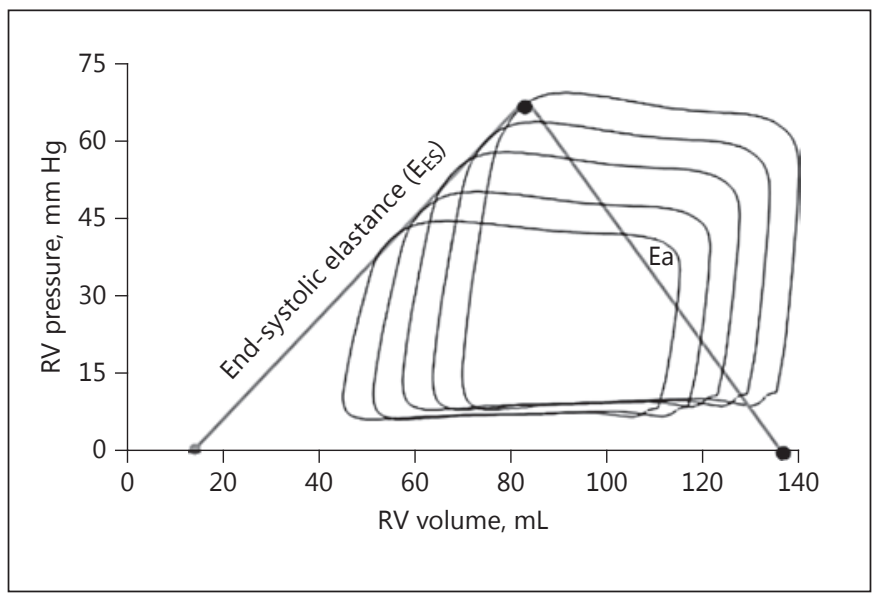

Fig. 4. Cartoon illustrating right ventricular (RV) pressure-volume loops at varying levels of preload decline. The slope of the line connecting the end-systolic pressure points is the end-systolic elastance $\left(\mathrm{E}_{\mathrm{ES}}\right)$, a load-independent measure of contractility. $\mathrm{E}_{\mathrm{ES}}$ can be compared with effective arterial elastance (Ea) to assess coupling of RV contractility to pulmonary vasculature load. Reproduced from Tedford [34] with permission from the publisher.

of the PAP decay curve by the occlusion method in order to better identify the site of predominant increased resistance in patients with CTEPH.

\section{Right Ventriculo-Arterial Coupling}

$\mathrm{PAH}$ is a disease that primarily affects the pulmonary vasculature; nevertheless, the symptomatology and outcome of patients with $\mathrm{PAH}$ are principally determined by right RV function [33, 43]. Furthermore, RV function is also a major independent prognostic factor in patients with postcapillary $\mathrm{PH}$, advanced respiratory diseases, and congenital heart diseases [44]. Accurate quantification of RV total hydraulic load by PVZ or by the simple formula presented in the previous section without taking into account the adaptation of the RV to the increased afterload is a simplification. Accordingly, the concept of a right ventricle-pulmonary circulation unit has been emerging that requires the simultaneous study of pulmonary circulation and RV function [45].

The pulmonary circulation presents a much lower resistance, greater compliance, and lower wave reflection than the systemic circulation; consequently, RV pressures are significantly lower than LV pressures under normal conditions [46]. RV adaptation to increased afterload is usually separated into two patterns: (1) homeometric (or 


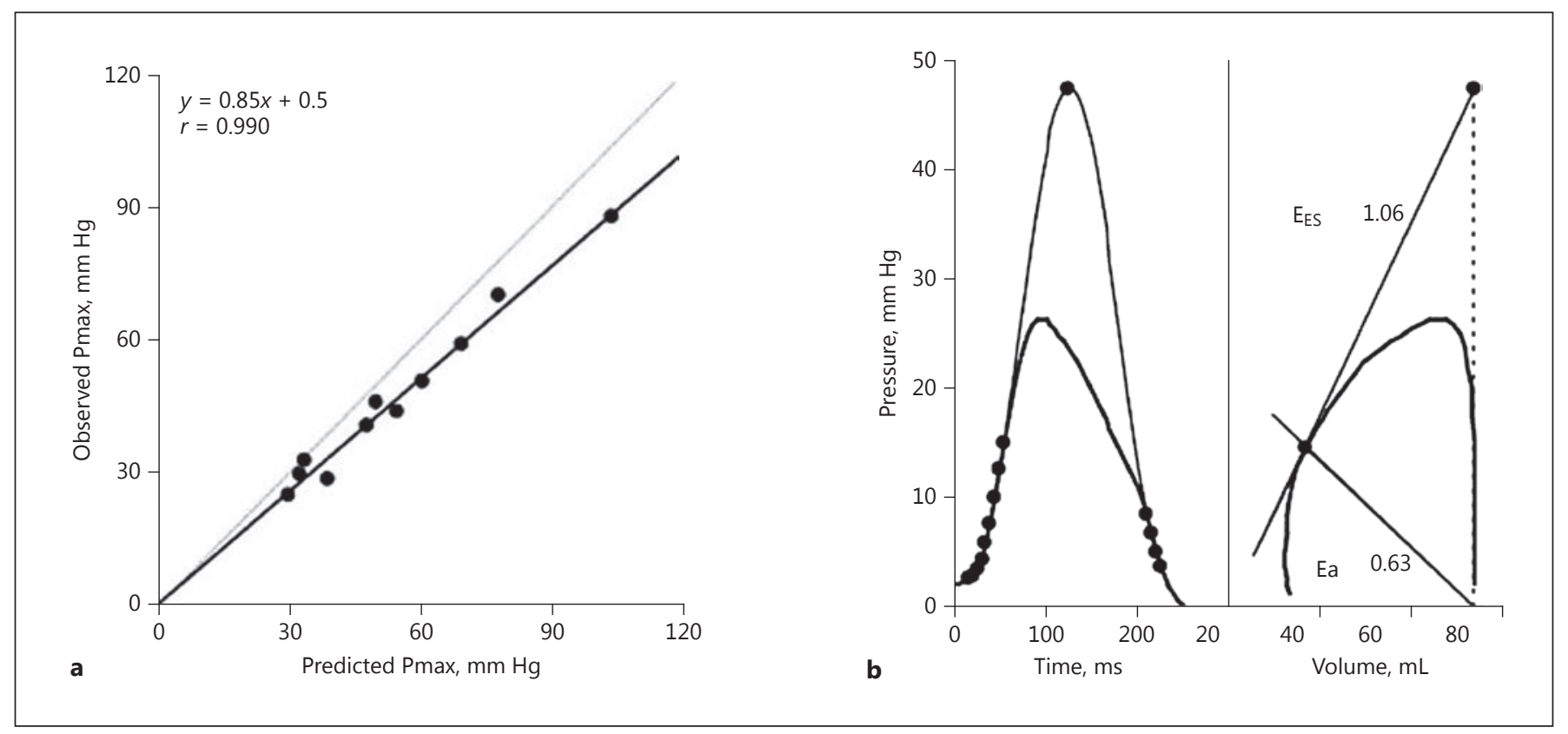

Fig. 5. Single-beat method for the measurement of right ventriculoarterial coupling in an anesthetized dog. a Good agreement between directly measured maximum right ventricular (RV) pressure (Pmax) when the pulmonary arterial trunk is clamped during 1 heart beat (black line) and the extrapolated Pmax (gray line). The slightly lower observed Pmax is explained by proximal pulmonary arterial compliance. b Pmax is calculated from early and late portions of the RV pressure curve, end-systolic elastance ( $\left.\mathrm{E}_{\mathrm{ES}}\right)$, or arterial elastance (Ea) graphically determined from Pmax and relative changes in volume and pressure during systole. Reproduced from Brimioulle et al. [51] with permission from the publisher.

pressure volume loop by dividing pressure at $\mathrm{E}_{\mathrm{ES}}$ by stroke volume, as presented in Figure 4. The impact of an elevated afterload on RV function can be assessed by computation of ventriculo-arterial coupling. Right ventriculo-arterial coupling is defined as the ratio of $\mathrm{E}_{\mathrm{ES}}$ to Ea. Optimal coupling occurs at $\mathrm{E}_{\mathrm{ES}} / \mathrm{Ea}$ ratios of 1.5-2, corresponding to the optimal energy transfer from the right ventricle to the pulmonary circulation [49].

This $\mathrm{E}_{\mathrm{ES}}$ and Ea determination is hampered by the requirement of instantaneous measurements of RV volumes and pressures with conductance catheters, which may be quite problematic given the complex geometry of the right ventricle. The single-beat approach, based on maximum pressure assessment from simple sinusoidal extrapolation of the early and late parts of an RV pressure curve, represents a valuable alternative to the ventricular pressure-volume loop for the assessment of right ventriculo-arterial coupling, as presented in Figure 5 [51]. Given the abovementioned difficulties in the simultaneous reliable determination of pressure and flow, right ventriculo-arterial coupling determinations have been reported only for a limited number of patients with $\mathrm{PH}$. Kuehne et al. [52] measured pressures and volumes by fluid-filled RHC and 
MRI, respectively, and assessed right ventriculo-arterial coupling by the single-beat method in patients with early iPAH, showing a 3-fold improvement in contractility consequent to an increased afterload with impaired right ventriculo-arterial coupling as compared with control subjects. Tedford et al. [53] measured RV pressures and volumes with conductance catheters and assessed $\mathrm{E}_{\mathrm{ES}}$ by a family of pressure-volume loops. Patients with systemic scleroderma-associated PAH had depressed coupling as compared with iPAH patients and systemic scleroderma patients without $\mathrm{PAH}$. The $\mathrm{E}_{\mathrm{ES}} / \mathrm{Ea}$ ratios were preserved in control subjects and in patients with chronic thromboembolic vascular disease without $\mathrm{PH}$, but they were reduced in patients with CTEPH [54]. Experimental and clinical studies on right ventriculo-arterial coupling suggest a predominant role of adaptive remodeling of the right ventricle confronted with an increased afterload at least in resting conditions. Maladaptive remodeling will first occur with a too high RV afterload maintained for a prolonged time or in the presence of a systemic disease [55].

Despite the fact that determination of right ventriculoarterial coupling represents the most accurate assessment of the impact of an elevated afterload on RV function, this technique nowadays is only feasible in the experimental setting. Due to recent and expected advancements in transthoracic echocardiography and in cardiac MRI, future research should be dedicated to developing invasive and, preferentially, noninvasive measures of coupling which can be easily used and integrated into routine clinical practice.

\section{Conclusions}

The pulmonary circulation in $\mathrm{PH}$ can be evaluated by simple or more complex methods, as presented in this narrative review. However, assessment of $\mathrm{PH}$ needs a multifaceted approach, and the clinical utility of combining different invasive hemodynamic methods still remains unclear, warranting further research. Single-point PVR determination cannot correctly assess the functional state of the pulmonary circulation, which is why multipoint pressure/flow coordinates are necessary. The very promising results with this approach should be confirmed in larger populations.

Nowadays, there is no reference method for assessing the operability of patients with CTEPH; consequently, the decision-making process remains complex. Analysis of the PAP decay curve by the occlusion method combined with calculation of the $\mathrm{RC}$ time seems a promising way to make a preoperative risk assessment regarding CTEPH and patient selection for medical therapy, but it still requires prospective validation in larger clinical trials. As an alternative to the technically demanding PVZ computation, RV afterload can be estimated from PAPm and mean $Q$ values by a simple formula in order to avoid instantaneous pulmonary pressure and flow determinations.

The pulmonary circulation and the right ventricle should no more be considered as two separate entities, but they should be viewed as a unique functional unit. New treatment options should ideally be evaluated by measurement of right ventriculo-arterial coupling in order to obtain a whole picture. This technique, however, is still immature for use at the bedside and in outpatient clinics. The pulmonary vasculature remains the primary target of medical therapy in PAH. However, novel therapeutic options aimed at preserving RV function as well as reversing RV dysfunction/failure are needed, since, overall, survival of PAH has modestly improved with the currently available $\mathrm{PAH}$-specific drugs but the long-term outcome remains poor as a consequence of progressive RV failure [56].

\section{References}

1 Hoeper MM, Bogaard HJ, Condliffe R, Franzt R, Khanna D, Kurzyna M, et al: Definitions and diagnosis of pulmonary hypertension. J Am Coll Cardiol 2013;62(suppl):D42-D50.

2 Galiè N, Humbert M, Vachiéry JL, Gibbs S, Lang I, Torbicki A, et al: 2015 ESC/ERS Guidelines for the diagnosis and treatment of pulmonary hypertension. The Joint Task Force for the Diagnosis and Treatment of Pulmonary Hypertension of the European Society of Cardiology (ESC) and the European Re- spiratory Society (ERS) endorsed by: Association for European Paediatric and Congenital Cardiology (AEPC), International Society of Heart and Lung Transplantation (ISHLT). Eur Heart J 2016;37:67-119.

3 Hatano S, Strasser T: Primary Pulmonary Hypertension, Report on a WHO Meeting. October 15-17, 1973. Geneva, World Health Organization, 1975.

4 Simonneau G, Gatzoulis MA, Adatia I, Celermajer D, Denton C, Ghofrani A, et al: Updat- ed clinical classification of pulmonary hypertension. J Am Coll Cardiol 2013;62(suppl): D34-D41.

5 Vachiéry JL, Adir Y, Barberà JA, Champion $\mathrm{H}$, Coghlan JR, Cottin V, et al: Pulmonary ypertension due to left heart diseases. J Am Coll Cardiol 2013;62(suppl):D100D108.

6 Rich JD, Rich S: Clinical diagnosis of pulmonary hypertension. Circulation 2014;130: $1820-1830$. 
7 Seeger W, Adir Y, Barberà JA, Champion H, Coghlan FG, Cottin V, et al: Pulmonary hypertension in chronic lung diseases. J Am Coll Cardiol 2013;62(suppl):D109-D116.

8 Lang IM, Madani M: Update on chronic thromboembolic pulmonary hypertension. Circulation 2014;130:508-518.

9 McGoon MD, Benza RL, Escribano-Subias P, Jiang X, Miller DP, Peacock AJ, et al: Pulmonary arterial hypertension: epidemiology and registries. J Am Coll Cardiol 2013;62(suppl): D51-D59.

10 Ling Y, Johnson MK, Kiely DG, Condliffe R, Elliot CA, Gibbs JS, et al: Changing demographics, epidemiology, and survival of incident pulmonary arterial hypertension: results from the pulmonary hypertension registry of the United Kingdom and Ireland. Am J Respir Crit Care Med 2012;186:790-796.

11 Kovacs G, Berghold A, Scheidl S, Olschewski $\mathrm{H}$ : Pulmonary arterial pressure during rest and exercise in healthy subjects: a systematic review. Eur Respir J 2009;34:888-894.

12 Lau EMT, Godinas L, Sitbon O, Montani D, Savale L, Jaïs X, et al: Resting pulmonary artery pressure of 21-24 $\mathrm{mm} \mathrm{Hg}$ predicts abnormal exercise haemodynamics. Eur Respir J 2016;47:1436-1444.

13 Barst RJ, McGoon M, Torbicki A, Sitbon O, Krowka MJ, Olschewski H, et al: Diagnosis and differential assessment of pulmonary arterial hypertension. J Am Coll Cardiol 2004; 43(suppl S):40S-47S.

14 Hervé P, Lau EM, Sitbon O, Savale L, Montani D, Godinas L, et al: Criteria for diagnosis of exercise pulmonary hypertension. Eur Respir J 2015;46:728-737.

15 Halpern SD, Taichman DB: Misclassification of pulmonary hypertension due to reliance on pulmonary capillary wedge pressure rather than left ventricular end-diastolic pressure. Chest 2009;136:37-43.

16 Paulus WJ, Tschöpe C, Sanderson JE, Rusconi C, Flachskampf F, Rademakers FE, et al: How to diagnose diastolic heart failure: a consensus statement on the diagnosis of heart failure with normal left ventricular ejection fraction by the Heart Failure and Echocardiography Associations of the European Society of Cardiology. Eur Heart J 2007;28:2539-2550.

17 LeVarge BL, Pomerantsev E, Channick RN: Reliance on end-expiratory wedge pressure leads to misclassification of pulmonary hypertension. Eur Respir J 2014;44:425-434.

18 Naeije R: Pulmonary vascular resistance. A meaningless variable? Intensive Care Med 2003;29:526-529.

19 Leeman M, Lejeune P, Closset J, Vachiéry JL, Mélot C, Naeije R: Nature of pulmonary hypertension in canine oleic acid pulmonary edema. J Appl Physiol (1985) 1990;69:293298.

20 Pagnamenta A, Fesler P, Vandinivit A, Brimioulle S, Naeije R: Pulmonary vascular effects of dobutamine in experimental pulmonary hypertension. Crit Care Med 2003;31: 1140-1146.
21 Castelain V, Chemla D, Humbert M, Sitbon O, Simonneau G, Lecarpentier Y, et al: Pulmonary artery pressure-flow relations after prostacyclin in primary pulmonary hypertension. Am J Respir Crit Care Med 2002;165: 338-340.

22 Provencher S, Hervé P, Sitbon O, Humbert M, Simonneau G, Chelma D: Changes in exercise haemodynamics during treatment in pulmonary arterial hypertension. Eur Respir J 2008; 32:393-398.

23 Lau EMT, Vanderpool RR, Choudhary P, Simmons LR, Corte TJ, Argiento P, et al: Dobutamine stress echocardiography for the assessment of pressure-flow relationships of the pulmonary circulation. Chest 2014;146:959966.

24 Ganter CG, Jakob M, Takala J: Pulmonary capillary pressure. Minerva Anestesiol 2006; 72:21-36.

25 Souza R, Amato MB, Demarzo SE, Deheinzelin D, Barbas CS, Schettino GP, Carvalho CR: Pulmonary capillary pressure in pulmonary hypertension. Crit Care 2005;9:R132-R138.

26 Fesler P, Pagnamenta A, Vachiéry JL, Brimioulle S, Abdel Kafi S, Boonstra A, et al: Single arterial occlusion to locate resistance in patients with pulmonary hypertension. Eur Respir J 2003;21:31-36.

27 Kim NHS, Fesler P, Channick RN, Knowlton KU, Ben-Yehuda O, Lee SH, et al: Preoperative partitioning of pulmonary vascular resistance correlates with early outcome after thromboendarterectomy for chronic thromboembolic pulmonary hypertension. Circulation 2004; 109:18-22.

28 Toshner M, Suntharalingam J, Fesler P, Soon E, Sheares KK, Jenkins D, et al: Occlusion pressure analysis role in partitioning of pulmonary vascular resistance in CTEPH. Eur Respir J 2012;40:612-617.

29 Gidwani UK, Mohanty B, Chatterjee K: The pulmonary artery catheter: a critical reappraisal. Cardiol Clin 2013;31:545-565.

30 Rosenkranz S, Preston IR: Right heart catheterisation: best practice and pitfalls in pulmonary hypertension. Eur Respir Rev 2015;24: 642-652.

31 Milnor WR: Hemodynamics, ed 2. Baltimore, Williams \& Wilkins, 1989.

32 Grant BJ, Lieber BB: Clinical significance of pulmonary arterial input impedance. Eur Respir J 1996;9:2196-2199.

33 Vonk-Noordegraf A, Haddad F, Chin KM, Forfia PR, Kawut SM, Lumens J, et al: Right heart adaptation to pulmonary arterial hypertension: physiology and pathobiology. J Am Coll Cardiol 2013;62(suppl):D22-D33.

34 Tedford RY: Determinants of right ventricular afterload (2013 Grover Conference series). Pulm Circ 2014;4:211-219.

35 Naeije R: Pulmonary circulation; in Grippi MA, et al (eds): Fishman's Pulmonary Diseases and Disorders, ed 5. McGraw Hill Medical, 2015, chapter 13 .
36 Pagnamenta A, Vanderpool R, Brimioulle S, Naeije R: Proximal pulmonary arterial obstruction decreases the time constant of the pulmonary circulation and increases right ventricular afterload. J Appl Physiol (1985) 2013;114:1586-1592.

37 Stergiopulos N, Meister JJ, Westerhof N: Evaluation of methods for estimation of total arterial compliance. Am J Physiol 1995; 268:H1540-H1548.

38 Lankhaar JW, Westerhof N, Faes TJ, Gan CT, Marques KM, Boonstra A, et al: Pulmonary vascular resistance and compliance stay inversely related during treatment of pulmonary hypertension. Eur Heart J 2008;29:16881695.

39 Saouti N, Westerhof N, Helderman F, Marcus JT, Stergiopulos N, Westerhof BE, et al: RC time constant of single lung equals that of both lungs together: a study in chronic thromboembolic pulmonary hypertension. Am J Physiol Heart Circ Physiol 2009;297:H2154$\mathrm{H} 2160$.

40 Saouti N, Westerhof N, Helderman F, Marcus JT, Boonstra A, Postmus PE, Vonk-Noordegraaf A: Right ventricular oscillatory power is a constant fraction of total power irrespective of pulmonary artery pressure. Am J Respir Crit Care Med 2010;182:1315-1320.

41 MacKenzie Ross RV, Toshner MR, Soon E, Naeije R, Pepke-Zaba J: Decreased time constant of the pulmonary circulation in chronic thromboembolic pulmonary hypertension. Am J Physiol Heart Circ Physiol 2013; 305:H259-H264.

42 Tedford RJ, Hassoun PM, Mathai SC, Girgis RE, Russell SD, Thiemann DR, et al: Pulmonary capillary wedge pressure augments right ventricular pulsatile loading. Circulation 2012;125:289-297.

43 Naeije R, Manes A: The right ventricle in pulmonary arterial hypertension. Eur Respir Rev 2014;23:476-487.

44 Haddad F, Doyle R, Murphy DJ, Hunt SA: Right ventricular function in cardiovascular disease, part II: pathophysiology, clinical importance, and management of right ventricular failure. Circulation 2008;117:1717-1731.

45 Champion HC, Michelakis ED, Hassoun PM: Comprehensive invasive and noninvasive approach to the right ventricle-pulmonary circulation unit: state of the art and clinical and research implications. Circulation 2009;120: 992-1007.

46 Gaine SP, Naeije R, Peacock AJ (eds): The Right Heart. London, Springer, 2014.

47 Campo A, Mathai SC, Le Pavec J, Zaiman AL, Hummers LK, Boyce D, et al: Hemodynamic predictors of survival in scleroderma-related pulmonary arterial hypertension. Am J Respir Crit Care Med 2010;182:252-260.

48 Mehra MR, Park MH, Landzberg MJ, Lala A Waxman AB; International Right Heart Failure Foundation Scientific Working Group: Right heart failure: toward a common language. J Heart Lung Transplant 2014;33:123126. 
49 Sagawa K, Maughan L, Suga H, Sunagawa K (eds): Cardiac Contraction and the PressureVolume Relationship. Oxford, Oxford University Press, 1988.

50 Vonk-Noordegraaf A, Westerhof N: Describing right ventricular function. Eur Respir J 2013;41:1419-1423.

51 Brimioulle S, Wauthy P, Ewalenko P, Rondelet B, Vermeulen F, Kerbaul F, Naeije R: Single-beat estimation of right ventricular endsystolic pressure-volume relationship. Am J Physiol Heart Circ Physiol 2003;284:H1625H1630.
52 Kuehne T, Yilmaz S, Steendijk P, Moore P, Groenink M, Saaed M, et al: Magnetic resonance imaging analysis of right ventricular pressure-volume loops: in vivo validation and clinical application in patients with pulmonary hypertension. Circulation 2004;110: 2010-2016.

53 Tedford RJ, Mudd JO, Girgis RE, Mathai SC, Zaiman AL, Housten-Harris T, et al: Right ventricular dysfunction in systemic sclerosisassociated pulmonary arterial hypertension. Circ Heart Fail 2013;6:953-963.
54 McCabe C, White PA, Hoole SP, Axell RG, Priest AN, Gopalan D, et al: Right ventricular dysfunction in chronic thromboembolic obstruction of the pulmonary artery: a pressurevolume study using conductance catheter. J Appl Physiol (1985) 2014;116:355-363.

55 Naeije R, Brimioulle S, Dewachter L: Biomechanics of the right ventricle in health and disease (2013 Grover Conference series). Pulm Circ 2014;4:395-406.

56 Galiè N, Palazzini M, Manes A: Pulmonary arterial hypertension: from the kingdom of the near-dead to multiple clinical trial metaanalyses. Eur Heart J 2010;31:2080-2086. 